\title{
Raman microstructural analysis of silicon-on-insulator formed by high dose oxygen ion implantation: As-implanted structures
}

\author{
J. Macía \\ Enginyeria i Materials Electrònics (EME), Departament de Física Aplicada i Electrònica, Universitat de \\ Barcelona, 08028 Barcelona, Spain \\ E. Martín \\ Departamento de Física de la Materia Condensada, Cristalografía y Mineralogía, Escuela Técnica \\ Superior de Ingenieros Industriales, 47011 Valladolid, Spain \\ A.Pérez-Rodríguez ${ }^{\mathrm{a})}$ \\ Enginyeria i Materials Electrònics (EME), Departament de Física Aplicada i Electrònica, Universitat de \\ Barcelona, 08028 Barcelona, Spain \\ J. Jiménez \\ Departamento de Física de la Materia Condensada, Cristalografía y Mineralogía, Escuela Técnica \\ Superior de Ingenieros Industriales, 47011 Valladolid, Spain \\ J. R. Morante \\ Enginyeria i Materials Electrònics (EME), Departament de Física Aplicada i Electrònica, Universitat de \\ Barcelona, 08028 Barcelona, Spain \\ B. Aspar and J. Margail \\ LETI (CEA-Technologies Avancées) Department Micro-Electronique, Centre d'Etudes Nucleaires de \\ Grenoble, 38041 Grenoble Cedex, France
}

(Received 26 March 1997; accepted for publication 15 July 1997)

\begin{abstract}
A microstructural analysis of silicon-on-insulator samples obtained by high dose oxygen ion implantation was performed by Raman scattering. The samples analyzed were obtained under different conditions thus leading to different concentrations of defects in the top Si layer. The samples were implanted with the surface covered with $\mathrm{SiO}_{2}$ capping layers of different thicknesses. The spectra measured from the as-implanted samples were fitted to a correlation length model taking into account the possible presence of stress effects in the spectra. This allowed quantification of both disorder effects, which are determined by structural defects, and residual stress in the top Si layer before annealing. These data were correlated to the density of dislocations remaining in the layer after annealing. The analysis performed corroborates the existence of two mechanisms that generate defects in the top $\mathrm{Si}$ layer that are related to surface conditions during implantation and the proximity of the top Si/buried oxide layer interface to the surface before annealing. (C) 1997 American Institute of Physics. [S0021-8979(97)07120-X]
\end{abstract}

\section{INTRODUCTION}

Silicon-on-insulator (SOI) has become an important alternative for the fabrication of integrated circuits (ICs) and advanced electronic devices in Si technology. ${ }^{1}$ SOI structures are formed by a top $\mathrm{Si}$ layer, which constitutes the active region where the devices are built up, and a buried $\mathrm{SiO}_{2}$ layer, which electrically isolates the active region from the Si substrate. This allows one to obtain dielectric isolation between adjacent devices in the circuit. These characteristics provide important advantages for ICs and devices fabricated on SOI structures over those made on bulk $\mathrm{Si}$, the main advantages being a higher density of integration, higher frequency of operation, and better resistance to radiation.

The main techniques for fabrication of SOI wafers are Separation by implanted oxygen (SIMOX), bonding and etch back SOI (BESOI), and SmartCut, ${ }^{1-3}$ respectively. SIMOX structures are very interesting for the development of submicron devices [metal-oxide-semiconductor (MOS) fully depleted devices]. For this, SOI wafers with very thin films (less than $100 \mathrm{~nm}$ ) can be fabricated by decreasing the im-

${ }^{\text {a)} E l e c t r o n i c ~ m a i l: ~ p e r e z-r o @ i r i s 1 . f a e . u b . e s ~}$ plantation energy and dose. ${ }^{4}$ Standard SIMOX wafers are formed by high dose oxygen ion implantation (with doses of the order of $10^{18} \mathrm{~cm}^{-2}$ ), followed by a high temperature anneal (at $1300{ }^{\circ} \mathrm{C}$ for several hours). This procedure allows one to prepare high quality SOI structures with top Si film thicknesses in the 200-300 $\mathrm{nm}$ range and buried oxide layer thicknesses in the 300-400 nm range. Four and six inch high quality wafers are commercially available. The fabrication of very thin film SOI structures by the BESOI technique, on the other hand, presents problems related to control of the etchback process. The main advantage of BESOI over SIMOX relates to the structural quality of the buried oxide layer, which is obtained by thermal oxidation. More recently, a new implantation based technique known as SmartCut was developed; ${ }^{3}$ this combines the advantages of both SIMOX (thickness control of the top Si film) and BESOI (control of both the thickness and structure of the buried oxide).

For ion implantation based technologies, a high temperature anneal is always required in order to relieve the structures from damage and residual stress in the top $\mathrm{Si}$ film. Implantation damage in the as-implanted top $\mathrm{Si}$ film is specially severe for SIMOX technology, where a very high dose 
of oxygen ions has to be implanted. Nondestructive characterization of this damage in terms of structural defects and stress is of great interest since both features determine the final defect density in the top layer (after annealing). Nondestructive techniques can, therefore, be used for on-line analysis of selected wafers in the fabrication process. Moreover, in-depth knowledge of the physical mechanisms related to the generation of defects in the structures is an important issue for further optimization of these technologies.

In this work, the structural analysis of SIMOX samples obtained under no standard conditions was performed by Raman scattering. The conditions were decided upon in order to obtain samples with different crystalline properties in the top silicon layer. This is possible by performing the implantation with the substrate capped with a thermal oxide layer (screen oxide). ${ }^{5}$ There are experimental proofs that relate the microstructure of the as-implanted layers and the density of defects remaining in the top Si layer after annealing to surface conditions during the implantation step since the characteristics of the surface determine its ability to accommodate the flux of interstitials coming from the oxidized region. ${ }^{5,6}$ The surface features therefore, dramically influence the solid phase epitaxial (SPE) regrowth rate at the surface.

For the as-implanted samples the first order Si Raman band appears broadened and frequency redshifted. ${ }^{7-9}$ Variations in the Raman parameters have been correlated to the presence in the top silicon layer of structural defects, such as precipitates and dislocations, as well as residual stress, all of which result from implantation induced damage. The structural defects reduce the lattice correlation length, which models the average distance between defects and causes disorder effects in the spectra. ${ }^{10}$ Analysis of the Raman spectra has allowed us to determine both the correlation length and the stress in the top Si layer of the as-implanted structures. These data were correlated to the remaining density of defects after annealing, and are discussed as a function of the main mechanisms involved in the generation of defects that are responsible for observed differences in the microstructure of the samples.

\section{EXPERIMENT AND SAMPLE DESCRIPTIONS}

Raman spectra were carried out with a Jobin-Yvon T64000 spectrometer coupled with an Olympus metallographic microscope. Both the excitation and collection of the scattered light were done through the microscope objective. Excitation was provided by the $457.9 \mathrm{~nm}$ line of an argon ionized laser, for which the penetration depth of scattered light is estimated to be about $140 \mathrm{~nm}$. The microscope objective used was $100 \times$, with a numerical aperture of 0.95 . Thus, the laser beam diameter at the focus is slightly submicronic according to the Rayleigh diffraction criterium. All the measurements were made at room temperature, and the laser power density was kept below $80 \mathrm{~kW} / \mathrm{cm}^{2}$ in order to avoid heating effects in the spectra. Under these conditions, the measurements were performed at a laser power density below threshold to induce temperature enhancement. ${ }^{11}$ All the measured spectra were systematically compared to those obtained on bulk silicon under the same experimental conditions.

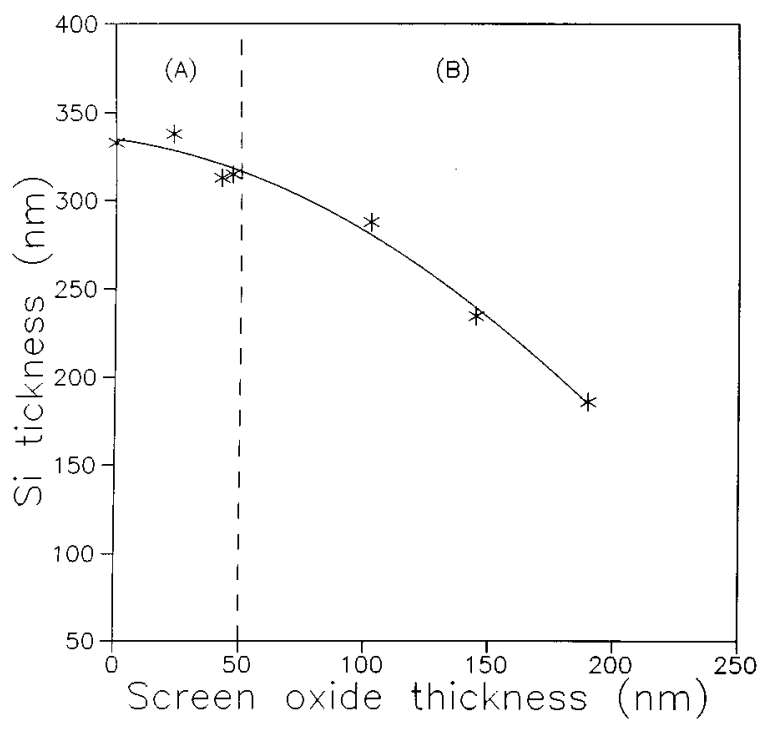

FIG. 1. Thickness of the top Si layer from as-implanted samples vs the screen oxide thickness. The thickness of the buried oxide layer is about 280 nm for all the samples.

All the SIMOX wafers studied here were implanted with a dose of $1.8 \times 10^{18} \mathrm{~cm}^{-2}$ oxygen ions, at an energy of 190 $\mathrm{keV}$, and implantation temperature of $T_{i}=650^{\circ} \mathrm{C}$. The $\mathrm{SiO}_{2}$ capping layer thickness was varied from 0 to $200 \mathrm{~nm}$. Henceforth this capping layer is refered to as the screen oxide. For each screen oxide thickness, two wafers were implanted: one wafer had no annealing (as implanted) and the other was annealed at $1320^{\circ} \mathrm{C}$ for $6 \mathrm{~h}$. The wafers analyzed in this work were grouped in two series: series A corresponds to wafers implanted with screen oxides thinner than $50 \mathrm{~nm}$, and series B corresponds to those implanted with screen oxides thicker than $100 \mathrm{~nm}$. As previously reported, ${ }^{5}$ for samples of series A the screen oxide is fully removed by surface sputtering during the implantation. For series $\mathrm{B}$ samples, the screen oxide is too thick to be fully removed during the implantation, and the whole implantation is performed with the surface covered with the capping oxide. Accordingly, the thickness of the top Si layer decreases as the screen oxide thickness increases. This can be seen in Fig. 1, where the thickness of the top $\mathrm{Si}$ layer of different as-implanted samples is plotted versus the screen oxide thickness. Besides,

TABLE I. Thickness of the screen oxide (SO) (in nm) and density of dislocations in the corresponding annealed samples (in $\mathrm{cm}^{-2}$ ) after Ref. 5. Series A corresponds to samples P02-P10, and series B corresponds to samples P12-P16.

\begin{tabular}{ccc}
\hline \hline Sample & $\begin{array}{c}d \\
(\mathrm{SO})\end{array}$ & $\begin{array}{c}\text { Dislocation } \\
\text { density }\end{array}$ \\
\hline P02 & 0 & $4 \times 10^{5}$ \\
P04 & 23 & $9 \times 10^{7}$ \\
P06 & 28.4 & $8 \times 10^{8}$ \\
P08 & 42.1 & $5 \times 10^{8}$ \\
P10 & 46.6 & $8 \times 10^{8}$ \\
P12 & 102 & $3 \times 10^{7}$ \\
P14 & 144.6 & $1.9 \times 10^{8}$ \\
P16 & 189.7 & $2 \times 10^{8}$ \\
\hline \hline
\end{tabular}




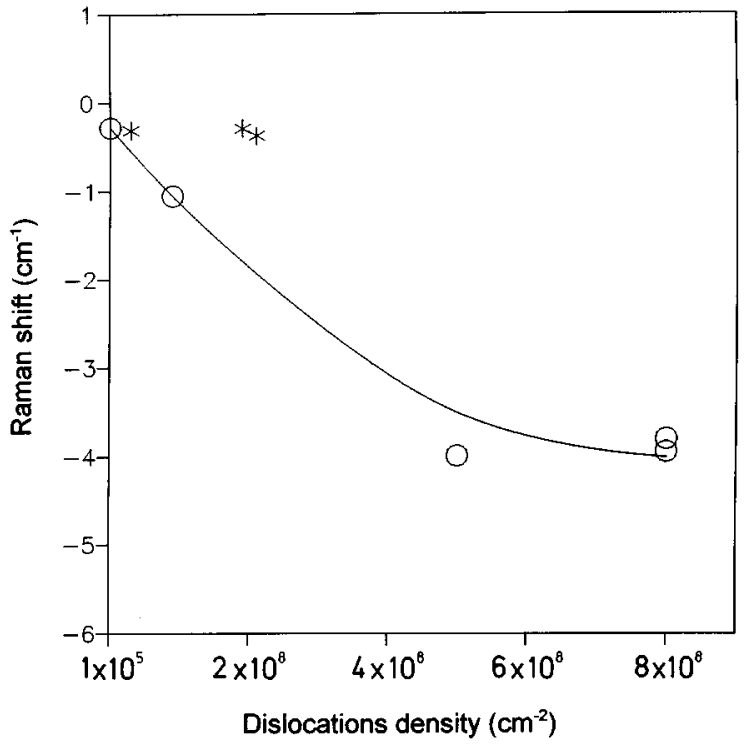

FIG. 2. Frequency shift of the Raman spectra of the as-implanted samples in relation to bulk silicon vs the dislocation density measured for the corresponding annealed ones: $(\bigcirc)$ series A and $(*)$ series B.

the density of dislocations in the top Si layer of corresponding annealed structures is reported in Table I. These data were determined by electron microscopy and enhanced chemical etching techniques. ${ }^{5}$ The data of Table I show that the dislocation density is strongly dependent on the implantation and surface conditions.

\section{RESULTS}

Figure 2 shows the frequency shift of the first order Raman band of the as-implanted samples with regard to the bulk silicon Raman frequency versus the dislocation density measured on the corresponding annealed samples. Figure 3

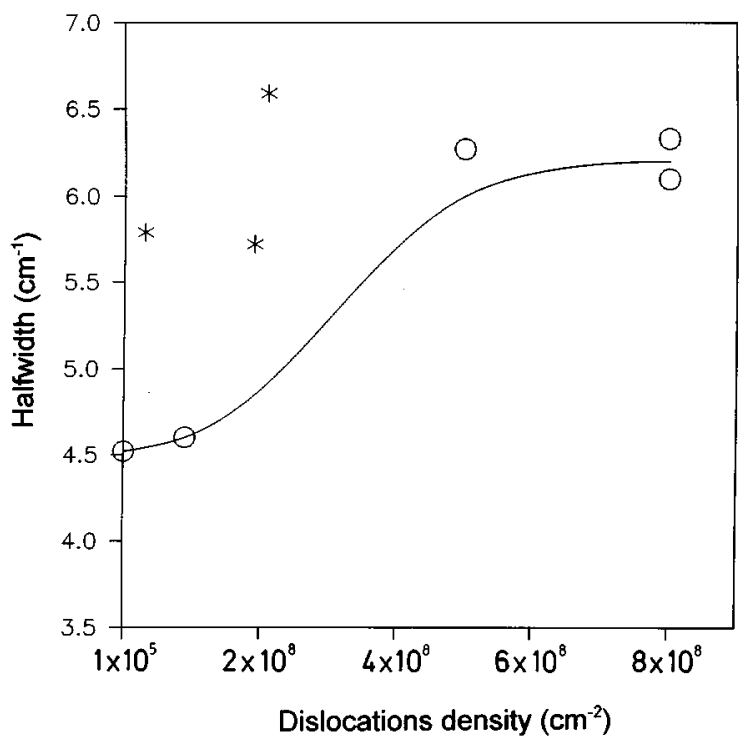

FIG. 3. FWHM of the Raman spectra of the as-implanted samples vs the dislocation density measured for the corresponding annealed ones: $(\bigcirc)$ series $\mathrm{A}$ and $(*)$ series $\mathrm{B}$. shows the full width at half-maximum (FWHM) of these spectra versus the dislocation density. The circles represent the samples implanted with the thinner screen oxide $(<50 \mathrm{~nm}$, samples of series A) and asterisks represent the samples implanted with the thicker screen oxides $(>100 \mathrm{~nm}$, samples of series B). Two different behaviors that can be associated with the two sample series can be observed.

(1) In the samples of series A the Raman parameters correlate to the final defect density. As the defect density increases, the Raman shift and FWHM increase as well, showing saturation for densities higher than $5 \times 10^{8} \mathrm{~cm}^{-2}$.

(2) In the samples of series B the Raman parameters do not show any correlation to the final defect density.

Since Raman measurements were carried out at very low laser power density, the reported spectral changes should be due to causes other than the asymmetric broadening associated with sample heating.

Since translational symmetry breakdown is expected in the as-implanted samples, which should result in a relaxation of the momentum conservation rule, the Raman spectra were studied in terms of a correlation length model. ${ }^{12}$ This model was initially developed by Nemanich and $\operatorname{Solin}^{13}$ and by Richter et al. ${ }^{14}$ and it has been applied to different systems in which phonon confinement effects are observed in the Raman spectra, such as micro- and nanocrystalline structures, ${ }^{15,16}$ ion implanted layers, ${ }^{17}$ and porous semiconductors. ${ }^{18,19}$ Assuming a constant correlation length $L$ in the scattering volume, the intensity of the first order Raman band of silicon is given by

$$
I(\omega) \propto \int_{0}^{2 \pi / a_{0}} \frac{|C(q)|^{2} 4 \pi q^{2} d q}{(\omega-\omega(q))^{2}+\left(\Gamma_{0} / 2\right)^{2}},
$$

where $a_{0}$ is the lattice constant of $\mathrm{Si}$, and $\Gamma_{0}$ is the Raman intrinsic linewidth of crystalline silicon.

The weight factor $C(q)$ for the scattering with wave vector $q$ is given by

$$
|C(q)|^{2} \propto \exp \left(-\frac{q^{2} L^{2}}{8}\right),
$$

and according to Ref. 18 the phonon dispersion relation $\omega(q)$ is taken as

$$
\omega(q)=\omega_{p}-120\left(\frac{q}{q_{0}}\right)^{2},
$$

where $\omega_{p}$ is the wave number of the first order Raman band in the absence of disorder effects and $q_{0}=2 \pi / a_{0}$. This relationship reproduces the dispersion relation for longitudinal optical (LO) phonons along the [001] in Si well. Taking into account that LO and transverse optical (TO) phonons in $\mathrm{Si}$ are degenerated at the zone center, and that for $L \gg a_{0}$ the phonons contributing to the integral (1) are mainly from the vicinity of the zone center, this expression constitutes a good approximation for $L \gg a_{0} .{ }^{18}$

Taking these correlations into account, we find that expressions (1)-(3) allow us to accurately fit the shape and position of the experimental spectra. However, the spectra are also affected by the presence of residual stress in the 
scattering volume. This stress can be taken into account by assuming a model in which both the correlation length and the residual stress are uniformly distributed in the scattering volume. ${ }^{10,16,19}$ In such a case, the stress does not affect the shape of the Raman spectra ${ }^{20}$ determined by expressions (1)-(3), and the only effect of stress is a shift of the spectra. According to this, the wave number of the first order Raman band in the absence of disorder effects, $\omega_{p}$, is shifted in relation to the wave number of the first order Raman line in the absence of both disorder and stress effects, $\omega_{0}$. This latter parameter corresponds to the position of the Raman line from stress free crystalline silicon $\left(521 \mathrm{~cm}^{-1}\right)$. Then, the stress induced shift is given by ${ }^{16}$

$$
\Delta \omega_{s}=\omega_{p}-\omega_{0} .
$$

So this shift corresponds to the difference between the position of the experimental spectrum and that of the theoretical spectrum from the correlation length model which reproduces the shape of the experimental one. ${ }^{19}$

Obviously in a layered structure like those studied here some depth distribution of both the correlation length and stress parameters must be present. Thus, the parameters estimated from the fitting of the spectra correspond to effective average values in the scattering volume. On the basis of an in plane stress model, we can estimate the magnitude of such stress to be proportional to the stress induced wave number shift $\Delta \omega_{s}:^{20}$

$$
\sigma(\mathrm{MPa})=250 \Delta \omega_{s}\left(\mathrm{~cm}^{-1}\right) .
$$

The fitting of the spectra with expressions (1)-(5) also has to consider the contribution to the spectrum from the $\mathrm{Si}$ substrate. In order to determine this contribution, we measured the Raman spectrum directly over the buried oxide once the top silicon layer was removed by a chemical treatment $(\mathrm{KOH})$. This spectrum was conveniently subtracted from the spectrum obtained in the full structures in order to apply the correlation length model without the substrate contribution. In general, an enhanced substrate contribution is observed for as-implanted SIMOX, which is likely related to a decrease of light absorption in the top Si layer due to the high oxygen content and oxide inclusions. ${ }^{10,21}$ Deconvolution of the Raman spectrum of sample P10, showing the contribution of both the top Si layer and the substrate, is presented in Fig. 4.

The stress induced frequency shift versus the thickness of the screen oxide is plotted in Fig. 5. Different behavior between the samples of the two series is observed: Samples of series A (thinner screen oxide) exhibit a shift towards the low frequency side for increasing screen oxide thickness. This means that tensile stress increases for increasing screen oxide thickness. On the other hand, samples of series B (thicker screen oxide) show compressive stress since a shift to high frequency is observed. For the latter series, the values of the stress induced shifts from the different samples are very similar within experimental accuracy, which suggests that stress is not significantly affected by the screen oxide thickness above a threshold value. Nevertheless, a certain increase of the compressive stress is observed as the screen oxide thickness increases.

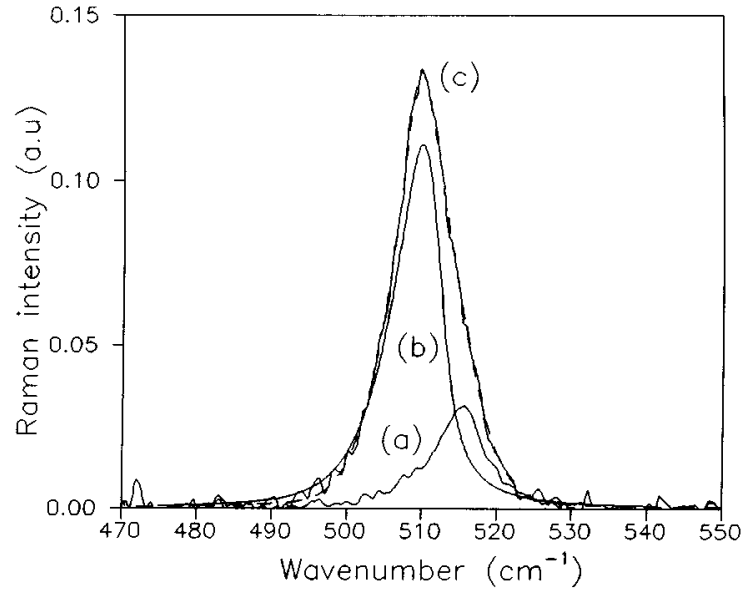

FIG. 4. Raman spectra from the P10 as-implanted sample: (a) substrate contribution, (b) theoretical spectrum from the top Si layer, (c) experimental (solid line) and theoretical (dashed line) spectrum of the whole structure.

The correlation length $L$ estimated from the theoretical fit of the spectra as a function of screen oxide thickness is plotted in Fig. 6. As is shown, the thicker the screen oxide, the shorter the correlation length. This also indicates that the thicker the screen oxide, the higher the defect density in the as-implanted samples. Moreover, for the samples of series A the correlation length decreases more significantly than that for the samples of series B.

\section{DISCUSSION}

The interpretation of these data has to take into account the different surface conditions during implantation for both series of samples. For the samples of series A, the screen oxide is completely removed by sputtering during implanta-

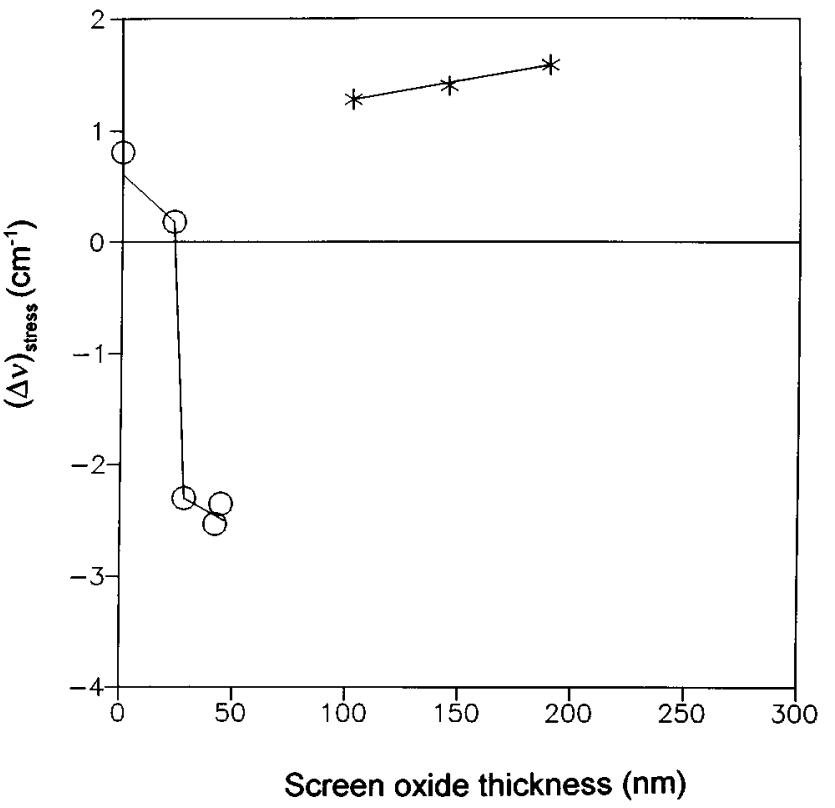

FIG. 5. Stress induced frequency shift vs screen oxide thickness: $(O)$ series $\mathrm{A}$ and $(*)$ series $\mathrm{B}$. 


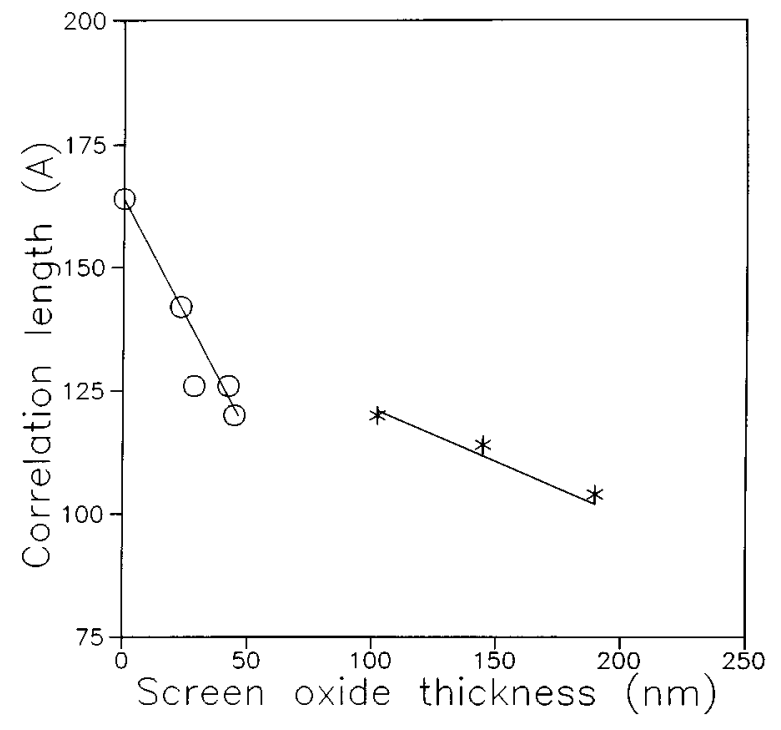

FIG. 6. Correlation length $L$ vs screen oxide thickness: $(\bigcirc)$ series A and (*) series B.

tion. This means that the implantation is performed over a surface free of screen oxide from the complete sputtering of the screen oxide to the end of the implantation. As the screen oxide thickness increases, this time decreases, and results in an increase of residual tensile stress. The tensile nature of this stress, in comparison with the compressive one observed for the samples of series B, suggests the presence of vacancies in the top Si layer of the samples of series A, whereas the compressive stress in the samples of series B may be associated with interstitial accumulation in the top layer. ${ }^{5}$

When the sample surface is partially free during implantation (series A), the excess of interstitials coming from the oxidized region relaxes by epitaxial regrowth during the time the surface is free. For samples with the thinnest screen oxide, the regrowth rate can achieve an acceptable level of ordering of the flux of silicon interstitials. However, as the thickness of the capping layer increases, the time available for an adequate epitaxial regrowth decreases. Then, before an atomic plan can be completed, the arriving silicon interstitials are placed on new atomic planes, leaving vacancies and increasing the tensile stress, in a way similar to that observed for amorphous Si layers.

For these samples there is a clear correlation between the density of defects in the as-implanted structures-monitored by the correlation length $L$-and the dislocation density in the corresponding annealed ones. This can be seen in Fig. 7, where $1 / L^{3}$ versus the dislocation density is plotted. The correlation between both magnitudes suggests that dislocations in the top Si layer after annealing are determined by the tensile stress and defects induced by the imperfect accommodation of $\mathrm{Si}$ interstitials at the surface.

In the samples from series $B$ the sputtering of the screen oxide layer during the implantation cannot remove this layer completely, and the silicon surface is covered by an oxide layer all during the implantation process. Accommodation of the silicon interstitials is then inhibited, and the interstitial concentration in the top silicon layer is very high. This ex-

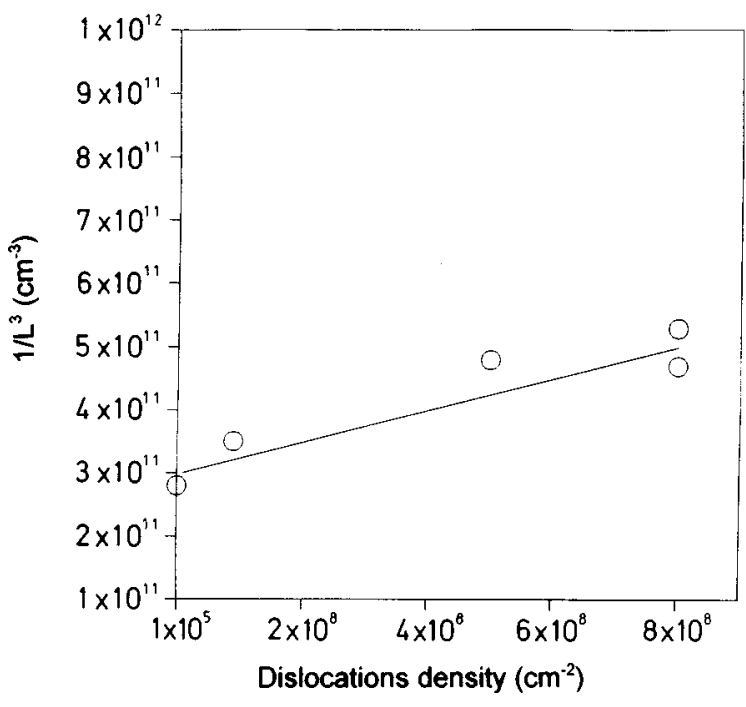

FIG. 7. 1/( correlation length $)^{3}$ vs the dislocation density measured for the corresponding annealed samples.

plains the compressive stress reported in Fig. 5. In this case compressive stress is the dominant effect, and it accounts for the frequency shift of the Raman peak to the blue in these samples.

The evolution of the density of dislocations in the top silicon layer after annealing can be explained as follows. ${ }^{5,22}$

(1) There is a sharp increase in the density of dislocations with the screen oxide thickness for the samples of series A. This is related to the sharp increase in the tensile stress and defects present in the surface region, resulting from imperfect epitaxial accommodation of interstitial $\mathrm{Si}$ in the surface. As the thickness of the screen oxide increases, the fraction of the implantation time with the surface of the sample free from the oxide decreases, which leads to poorer relaxation of excess interstitials in the surface region that determines the observed increase of tensile stress and the shortening of the correlation length.

(2) For the samples of series B, the increase in the dislocation density with the thickness of the screen oxide is smaller. This is related to the defects in the lower part of the top silicon layer from the as-implanted material that generate threading dislocations after annealing. The increase of the dislocation density is due to the displacement of this region towards the silicon surface as the screen oxide thickness increases. Similar results have been observed by changing the implantation energy and/or dose. ${ }^{22}$

In this last case, the correlation length and stress induced shift present weaker dependencies on the thickness of the screen oxide. This indicates that changes in the structure are smaller. The main difference between these samples is the decrease of the thickness of the top silicon layer as the thickness of the screen oxide increases. This lends support to mechanism (2) proposed for the generation of defects in these samples. 
Finally, we have to note the correlation observed between the Raman features of the as-implanted structures and the final density of dislocations after annealing for series A samples (as shown in Figs. 2 and 3). Similar behavior has been observed for samples implanted under different conditions without the screen oxide and with final dislocation densities above $5 \times 10^{4} \mathrm{~cm}^{-2}$. ${ }^{10}$ The Raman spectra from the as-implanted samples are thus sensitive to levels of damage in the structures that lead to final defect densities higher than this value after annealing. For defect densities higher than about $5 \times 10^{8} \mathrm{~cm}^{-2}$, however, the Raman parameters tend to saturate. This indicates that Raman scattering can be used before the annealing stage to detect, in a nondestructive way, wafers that after annealing will have a concentration of defects higher than $5 \times 10^{4} \mathrm{~cm}^{-2}$, and can also be used to estimate the final concentration for values in the range 5 $\times 10^{4}-5 \times 10^{8} \mathrm{~cm}^{-2}$.

\section{CONCLUSIONS}

The analysis performed shows the ability of Raman spectroscopy for the structural assessment of multilayer SOI structures. The spectra measured from as-implanted samples obtained under specific surface conditions-leading to very different dislocation densities after annealing-were interpreted in terms of the correlation length model. This has allowed us to deconvolute the spectra from the disorder effects related to the presence of defects and residual stress in the layers, as well as to estimate effective values of the correlation length and stress parameters. The results obtained show the strong influence of the surface conditions during implantation on the residual stress and the density of defects: the shorter the time the surface is free the higher the tensile stress and the density of defects. This is likely related to the surface having a poorer ability to accommodate excess interstitial Si coming from the oxidized regions. For samples implanted with a thicker screen oxide, residual compressive stress due to the accumulation of interstitials in the top layer is observed. Both the compressive stress and the correlation length show weaker dependencies on the screen oxide thickness. In this case, the increase of the density of dislocations after annealing is determined by the shift of the top Si/buried oxide interface towards the surface. Finally, the correlation observed between the Raman features of the as-implanted structures of series A and the final density of defects in the corresponding annealed wafers corroborates the ability of this nondestructive technique to diagnose the final density of defects in annealed wafers from measurements on asimplanted ones when the final density is higher than 5 $\times 10^{4} \mathrm{~cm}^{-2}$.

\section{ACKNOWLEDGMENTS}

This work was partially funded by the Spanish CICYT under Projects Refs. MAT92-0351 and MAT94-0042.

${ }^{1}$ Silicon-On-Insulator Technology and Devices, The Electrochemical Society Proceedings Vol. 94-11, edited by S. Cristoloveanu (The Electrochemical Society, Pennington, NJ, 1994).

${ }^{2}$ K. Izumi, Vacuum 42, 333 (1991).

${ }^{3}$ M. Bruel, Electron. Lett. 31, 1201 (1995).

${ }^{4}$ L. F. Giles, N. Meyyappan, A. Nejim, J. Blake, F. Cristiano, and P. L. F. Hemment, in Ref. 1, p. 50.

${ }^{5}$ J. Margail, J. M. Lamure, and A. M. Papon, Mater. Sci. Eng. B 12, 27 (1992).

${ }^{6}$ J. Stoemenos, K. J. Reeson, A. K. Robinson, and P. L. F. Hemment, J. Appl. Phys. 69, 793 (1991).

${ }^{7}$ A. Pérez-Rodríguez, A. Cornet, J. R. Morante, J. Jiménez, P. L. F. Hemment, and K. P. Homewood, J. Appl. Phys. 70, 1678 (1991).

${ }^{8}$ J. Takahashi and T. Makino, J. Appl. Phys. 63, 87 (1988).

${ }^{9}$ D. J. Olego, H. Baumgart, and C. K. Celler, Appl. Phys. Lett. 52, 483 (1988).

${ }^{10}$ J. Macía, T. Jawhari, A. Pérez-Rodríguez, and J. R. Morante, in Ref. 1, p. 148.

${ }^{11}$ E. Martín, J. Jiménez, A. Pérez-Rodríguez, and J. R. Morante, Inst. Phys. Conf. Ser. 135, 105 (1994).

${ }^{12}$ P. M. Fauchet and I. H. Campbell, CRC Crit. Rev. Solid State Mater. Sci. 14, S79 (1988).

${ }^{13}$ R. J. Nemanich and S. A. Solin, Phys. Rev. B 20, 392 (1979).

${ }^{14}$ H. Richter, Z. P. Wang, and L. Ley, Solid State Commun. 39, 625 (1981).

${ }^{15}$ Z. Iqbal and S. Veprek, J. Phys. C 15, 377 (1982).

${ }^{16}$ H. Xia, Y. L. He, L. C. Wang, W. Zhang, X. N. Liu, X. K. Zhang, and D. Feng, J. Appl. Phys. 78, 6705 (1995).

${ }^{17}$ K. K. Tiong, P. M. Amirtharaj, F. H. Pollak, and D. E. Aspnes, Appl. Phys. Lett. 44, 122 (1984).

${ }^{18}$ Z. Sui, P. P. Leong, I. P. Herman, G. S. Higashi, and H. Temkin, Appl. Phys. Lett. 60, 2086 (1992).

${ }^{19}$ M. Yang, D. Huang, P. Hao, F. Zhang, X. Hou, and X. Wang, J. Appl. Phys. 75, 651 (1994).

${ }^{20}$ E. Anastassakis, Physical Problems in Microelectronics, Proceedings of the 4th International School ISPPM, Varna, Bulgaria, 1985, edited by J. Kassavod p. 128.

${ }^{21}$ J. Macía, Ph.D. thesis, University of Barcelona, 1996.

${ }^{22}$ J. Stoemenos, B. Aspar, and J. Margail, in Ref. 1, p. 16. 